\title{
Effects of Pore Design on Mechanical Properties of Nanoporous Silicon
}

\author{
Nicholas Winter, Matthew Becton, Liuyang Zhang, and Xianqiao Wang ${ }^{*}$ \\ College of Engineering, University of Georgia, Athens, GA 30602 \\ "Corresponding author: xqwang@uga.edu
}

\begin{abstract}
Nanoporous silicon has been emerging as a powerful building block for next-generation sensors, catalysts, transistors, and tissue scaffolds. The capability to design novel devices with desired mechanical properties is paramount to their reliability and serviceability. In order to bring further resolution to the highly variable mechanical characteristics of nanoporous silicon, here we perform molecular dynamics simulations to study the effects of ligament thickness, relative density, and pore geometry/orientation on the mechanical properties of nanoporous silicon, thereby determining its Young's modulus, ultimate strength, and toughness as well as the scaling laws versus the features of interior ligaments. Results show that pore shape and pattern dictate stress accumulation inside the designed structure, leading to the corresponding failure signature, such as stretching-dominated, bending-dominated, or stochastic failure signatures, in nanoporous silicon. The nanostructure of the material is also seen to drive or mute size effects such as "smaller is stronger" and "smaller is ductile". This investigation provides useful insight into the behavior of nanoporous silicon and how one might leverage its promising applications.
\end{abstract}

Keywords: Nanoporous materials, Scaling law, Failure, Molecular dynamics 


\section{Introduction}

The design and fabrication of nanoporous materials are hot topics at the forefront of current materials science. With the availability of advanced nanoscopic design techniques including ion etching [1], chemical vapor deposition [2], and atomic manipulation via scanning tunneling electron microscopy [3], tailoring nanostructures to a specific design is not only possible, but the fabrication processes to achieve these nanostructures continue to increase in precision and viability. Nanopores bestow incredible characteristics upon materials such as high surface-tovolume ratios, increased catalytic power [4], high yield strength [5], and strain reversibility [6]. These properties make nanoporous materials ideal for applications in sensing [7], tissue engineering [8], fuel cell technology [9], and sequestration/filtration [10].

Nature seems to have mastered the design of nanostructured materials and devices through the iterative processes of natural selection, for example bone which is hard and strong under compressive force yet light weight due to its porous structure, spider silk with a unique combination of high tensile strength, extensibility, and flexibility that derived from peptide bonds and simple hydrogen bonds, and the diatom's intricate cell-wall, which is a mechanically superb, nanoporous structure composed of amorphous silica [11] as shown in Figure 1(a) [12]. The pores in the diatom's cell-wall create remarkably uniform designs at the nanoscale ranging from ribs to honeycombs [11]. These designs have been shown to heavily influence the mechanical properties of the diatom's cell-wall [13] and represents the larger end of the scale for $\mathrm{np}$-Si with pore sizes reaching up to $\sim 1500 \mathrm{~nm}$ (Figure 1(b)). At the smallest end of the scale is MCM-41 (Mobil Composition of Matter No. 41, a family member of np-Si materials) with nanopores as small as 2nm Figure 1(c) [14], which provides a realistic justification for our computational model in the Section 2. Nanoporous silicon with pore size range from $2 \mathrm{~nm}$ to 
$1,500 \mathrm{~nm}$ has been widely used in industrial applications including the connection of catalytic components such as enzymes [15], anodes in lithium ion batteries due to its high energy density [16], resistive random-access memory in computers (RRAM) [17], and scaffolding for cell encapsulation and implantation due to the well-understood saline chemistries as well as its anisotropic mechanical properties [8]. np-Si has also been emerging as a fuel in microelectromechanical systems because it is highly energetic and its rate of energy release is tunable [9], and has also shown promise as a highly-sensitive ion-selective transistor owing to its conductivity and very high surface area to volume ratio [7].

Understanding how the mechanical properties of np-Si may be altered is important for designing $\mathrm{np}-\mathrm{Si}$ for the previously stated applications. For instance, one promising technique in RRAM is electromechanical coupling using mechanical strains to induce a change in the magnetic order of a material and is a possible solution for decreasing the energy needed to store information[17]. The magnetic order of $\mathrm{np}-\mathrm{Si}$ in this scenario is dependent on the absolute strain that it is experiencing and therefore the material's elastic modulus is an important design parameter for sizing the np-Si and developing np-Si that satisfies the engineering constraints. In previous studies by Garcia et al. tension studies on nanoporous silica with a rectangular mesh geometry and constant void volume between various sized models have been simulated. They found that the failure mechanism of nanoporous silica is correlated with relative density and ligament thickness and can change from crack propagation dominated failure, to failure characterized by the competition between crack propagation and shear mechanisms, and even failure fitting the criteria of ductile rupture $[18,19]$. Garcia et al. also claim that as ligament size is decreased the ductility of nanoporous silica increases, its elastic modulus decreases, its strength decreases, and that its toughness has a complex relationship with ligament thicknesses below 27 angstroms [18- 
20]. These studies shed light on the nature of the connection between mechanical properties of nanoporous silicon and size but by maintaining a constant void volume while changing the size of ligaments the relative density must be changed too and so the observations cannot conclude whether the cause of changes in mechanical response is due to relative density or size effects. To further these investigations, we tease apart the effects of relative density and ligament thickness to make clear how each design parameter changes the mechanical response of nanoporous silicon and we also test the effects of pore geometry on the mechanics of nanoporous silicon as well. To better understand how one might manipulate the mechanical properties of np-Si via control of its nanoporous structure, here we computationally model np-Si with various relative densities, ligament thicknesses, and pore geometries under tensile loadings and discuss the results.

\section{Computational Models and Methods}

For this work, molecular dynamics simulations based on the open-source code LAMMPS are performed in order to investigate the mechanical behavior of $\mathrm{np}-\mathrm{Si}$ [21]. Through preliminary stress simulations (see Figure S1 in the Supplemental Materials) on bulk silicon using the environmentally dependent interatomic potential (EDIP) [22], Stillinger-Weber (SW) Potential [23], and Tersoff potential [24], we found that the Stillinger-Weber potential shows the best accuracy in bulk modulus and elastic modulus with comparison of the experimental data stressstrain curves [25]. Therefore, we choose the SW potential to perform the relevant MD simulations. The details of SW potential are tabulated as follows:

$$
\begin{gathered}
E=\sum_{i} \sum_{j>i} \varphi_{2}\left(r_{i j}\right)+\sum_{i} \sum_{j \neq i} \sum_{k>j} \varphi_{3}\left(r_{i j}, r_{i k}, \theta_{i j k}\right) \\
\varphi_{2}\left(r_{i j}\right)=A_{i j} \epsilon_{i j}\left[B_{i j}\left(\frac{\sigma_{i j}}{r_{i j}}\right) p_{i j}-\left(\frac{\sigma_{i j}}{r_{i j}}\right) q_{i j}\right] e^{\left(\frac{\sigma_{i j}}{r_{i j}-a_{i j} \sigma_{i j}}\right)}
\end{gathered}
$$




$$
\varphi_{3}\left(r_{i j}, r_{i k}, \theta_{i j k}\right)=\lambda_{i j k} \epsilon_{i j k}\left[\cos \theta_{i j k}-\cos \theta_{o i j k}\right]^{2} e^{\left(\frac{\gamma_{i j} \sigma_{i j}}{r_{i j}-a_{i j} \sigma_{i j}}\right)} e^{\left(\frac{\gamma_{i k} \sigma_{i k}}{r_{i k}-a_{i k} \sigma_{i k}}\right)}
$$

where $\varphi_{2}$ is a two-body term describing the interaction of two atoms (ex: $\left.\mathrm{Si}_{1} \mathrm{Si}_{2}\right)$ and $\varphi_{3}$ is a three-body term representing the interaction of three atoms (ex: $\mathrm{Si}_{1} \mathrm{Si}_{2} \mathrm{Si}_{3}$ ). The summations in the formula are over all neighbors $\mathrm{J}$ and $\mathrm{K}$ of atom $i$ within a cutoff distance equal to $a \times \sigma$.

Here, models with dimensions $43 \mathrm{~nm} \times 43 \mathrm{~nm} \times 7 \mathrm{~nm}$, inspired by the thin nanoporous silicon plate shown in Figure 1(c) $[12,14,26]$, are used to study the mechanical properties and failure patterns of np-Si materials. In order to investigate the effect of pore patterns on the mechanical properties of $\mathrm{np}-\mathrm{Si}$, we propose three different pore patterns under the same relative density: staggered circles (circles), staggered horizontal ellipses (H-ellipses, whose major axis is aligned with the loading direction), and staggered vertical ellipses (V-ellipses, whose major axis is orthogonal to the loading direction), as shown in Figure 2(a) (the elliptical major axes range in size from $3.5 \mathrm{~nm}$ to $34.3 \mathrm{~nm}$ while the minor axes range from $1.9 \mathrm{~nm}$ to $18.2 \mathrm{~nm}$ and the circle diameters range from $1.9 \mathrm{~nm}$ to $26.1 \mathrm{~nm}$, all ellipses have an eccentricity $\sim 0.8$ ). Circular and elliptical pores ae chosen in this study because they are common motifs in manufactured/natural materials examples (see examples in Figures S2, S3 and S4 of the Supplemental Materials). Of equal importance to the mechanical properties of porous materials is their relationship with relative density therefore we propose a set of models for each porous geometry with various relative densities with an example shown in Figure 2(b) for H-ellipse np-Si. In addition to pore pattern and relative density, ligament size has been shown to play an important role in the mechanical behaviors of other np-materials such as np-gold [27] and np-aluminum [28], giving us reason to investigate the effect of ligament size on the mechanical properties of np-Si as well with representative models shown in Figure 2(c). Here, models with dimensions of $43 \mathrm{~nm} \times$ 
$43 \mathrm{~nm} \times 7 \mathrm{~nm}$, inspired by the thin, two-dimensional, porous-plate $\mathrm{np}-\mathrm{Si}$ structures shown in Figure $1[12,14,26]$, are used to study the mechanical properties and failure patterns of np-Si materials with constant ligament thickness and variable relative density as shown in Figure 2(b). While computational models used to study effect of ligament size on the mechanical properties of $\mathrm{np}-\mathrm{Si}$ are $7 \mathrm{~nm}$ thick in the $z$-direction with the $x$-and $y$-axes lengths varying from $9.5 \mathrm{~nm} \times$ $9.5 \mathrm{~nm}$ to $73 \mathrm{~nm} \times 73 \mathrm{~nm}$ shown in Figure 2(c). For the purpose of clarity, we define the relative density, $\bar{\rho}=\frac{\rho_{*}}{\rho_{b}}$ of the models as the ratio of the density of the chosen porous model, $\rho_{*}$, to the density of the bulk material, $\rho_{b}$. Also, ligament thickness is measured as the average width of npSi struts in the $x y$-plane where adjacent pores are closest together.

All simulations are performed using the standard velocity-Verlet time integration with a time step of $1 \mathrm{fs}$. Periodic boundary conditions are applied in the $x, y$, and $z$ directions in order to mimic a specimen with an infinite size. Meanwhile, from the viewpoint of computational modeling in order to avoid the self-interaction among atoms due to the periodic boundary condition while preserving computational efficiency, we choose the model with $7 \mathrm{~nm}$ thick in the z-direction. At the beginning of each simulation an energy minimization based on the conjugate gradients method is imposed on the np-Si models. During the energy minimization process the boundaries of the np-Si models are allowed to expand or contract freely and afterwards a canonical isothermal-isochoric ensemble (NVT) using a Nose-Hoover thermostat is performed for 25 picoseconds to thermally equilibrate the np-Si. In order to mitigate the initial residual stresses in the np-Si model, an isothermal-isobaric ensemble (NPT) is invoked for another 25 picoseconds. Following the energy minimization and thermal/baric equilibrations we apply a tensile load to the np-Si model with a strain rate of $0.05 / n s$ in the $\left[\begin{array}{lll}1 & 0 & 0\end{array}\right]$ direction. All visualizations of the models are constructed from the OVITO software[29]. For stress analysis, 
here two types of stress are used: one is the overall stress or average stress with a unit of GPa which is used to present the stress-strain relationship; the other is per-atom stress with a unit of $G P a \times n m^{3}$ which is used to present the stress distribution inside the model.

\section{Results and Discussions}

We now analyze the effects of pore geometry, relative density, and ligament diameter on the mechanical properties of np-Si. Bulk silicon is inherently brittle and has a modest strength, $\mathrm{np}-\mathrm{Si}$ may tend to be brittle and not impressively strong however the behavior of the np-Si material is strongly influenced by its nanoscopic geometry and fits into viable strength to weight ratio ranges for electrical engineering [16], tissue engineering [8], and chemical engineering applications [17].

\subsection{Effects of pore designs on stress concentration and material failure}

In relevant studies, pore design is shown to affect the mechanical behaviors such as stiffness, strength, and recoverability of porous materials including porous polymers [30], hydroxyapatite bioceramics [31], and aluminum oxide ceramics [32]. However, there are few reports on using pore design in np-Si to achieve a specific mechanical property. Therefore, it is interesting to look into the effect of pore geometry on np-Si materials by means of computational modeling. Figure 3 here shows a side by side comparison of three np-Si models (staggered circles, H-ellipses, and V-ellipses) with different relative densities but the same ligament thicknesses of $d=3.5 \mathrm{~nm}$. All models are colored by per atom stress in the loading direction $\left[\begin{array}{lll}1 & 0 & 0\end{array}\right]$ and each color gradient has the same range ( 0 to $0.35 \mathrm{GPa} \times \mathrm{nm}^{3}$ ). The first row in Figure 3 shows the stress concentration and distribution in each model at its peak loading capacity without any damage inside the structure. The proceeding snapshots capture the failure of each model and show that each np-Si design experiences ligament failure initiated by competing mechanisms, shearing and crack 
propagation, and completed by ligaments necking to ductile rupture. The distribution and concentration of stress for each atom within each structure can be observed further in Figures 4(a), 4(b), and 4(c), where each model is pictured at the same loading (848.2 $\mathrm{nN})$ and only atoms experiencing a per atom stress over $0.35 \mathrm{GPa} \times \mathrm{nm}^{3}$ are shown. With the ligament thickness and strain rate being kept fixed, pore geometry becomes the major design variable and Figure 4 clearly shows that the mechanical behavior of np-Si with H-ellipses allows stresses to distribute evenly throughout the structure (Figure 4(b)) with a low average per atom stress of $0.158 \mathrm{GPa} \times \mathrm{nm}^{3}$ (obtained from the stress distribution curve Figure 4(d)). With per atom stresses being distributed evenly and remaining comparatively low, np-Si with H-ellipses shows great strength and a high elastic modulus with respect to the other two models as shown in Table 1. Once pore geometry is changed to staggered circles, stresses become more concentrated within the structure raising the average per atom stress to $0.176 \mathrm{GPa} \times \mathrm{nm}^{3}$. With the average stress climbing (driven by the number of atoms able to carry large stresses falling) the elastic modulus and strength of np-Si are effectively lowered. The concentration of stresses increases further after changing the orientation of the elliptical pattern from H-ellipses to V-ellipses. The stresses in the V-ellipse np-Si are highly concentrated; the average per atom stress reaches 0.183 $G P a \times n m^{3}$ within this structure resulting in a further decrease of ultimate strength and elastic modulus. Figure S5 in the Supplemental Materials shows that there is no consistent pattern for the stress-strain relationship among these three different geometries even if stress is normalized by density of the samples.

An overview of the effect of pore geometry on the mechanical properties can be seen in Table 1 where several mechanical properties of np-Si models with ligament thicknesses of $3.5 \mathrm{~nm}$ are shown. From it can be noticed that how Young's modulus, ultimate strength and toughness of 
$\mathrm{np}-\mathrm{Si}$ are dramatically affected by changing the pore geometry and pore orientation, while keeping ligament size unchanged. For example, the H-ellipse np-Si can have an elastic modulus that is slightly greater than staggered circle np-Si, 1.2 times greater, but much greater than $\mathrm{V}$ ellipse np-Si at 2.7 times. In contrary, although strength and elastic modulus are the lowest in npSi with V-ellipses, it has a toughness that is higher than that of np-Si with staggered circles showing that changing the nanostructure does not always induce a monotonic increase or decrease in all mechanical properties; in fact this can be used to tune materials for specific functions.

\subsection{Effects of relative density}

The mechanical properties and scaling laws of macroporous materials and np-materials have been shown to be dependent on pore geometry [30,31], ligament thickness [33, 34], relative density [34, 35], and grain size [36-38]. Experimental and computational studies also reveal that strength and elastic moduli of np-materials scale differently than microporous and macroporous materials with respect to relative density [34, 35, 39]. Figure 5 shows the uniaxial stress-strain curves for np-Si structures with constant ligament diameters but variable relative densities. Upon the initiation of deformation, the stress builds up linearly until the material yields. After reaching the ultimate strength, $\sigma_{u}$, the stress decreases with increasing strain as the material's ligaments begin to tear and rupture. The elastic modulus $E$, the ultimate strength $\sigma_{u}$, and the toughness $\kappa$ of a given np-Si model are measured from the stress-strain curves and used to develop scaling laws.

The deformation of cellular solids can be dominated by bending or stretching; the failure regime is dictated by the geometry of the lattice and its connectivity. The strength and elastic modulus of an ideal stretching-dominated structure scale linearly with relative density $E \sim \bar{\rho}$ and $\sigma_{u} \sim \bar{\rho}$ [40] 
while bending-dominated and stochastic architectures scale as $E \sim \bar{\rho}^{2}$ and $E \sim \bar{\rho}^{3}$ respectively [41]. The yield strength and modulus of 3D open-cell bending dominated structures, such as honeycombs or octahedral lattices, scale as $\sigma_{u}=0.3 \sigma_{b} \bar{\rho}^{1.5}$ and $E=E_{b} \bar{\rho}^{2}[42,43]$. np-Si silicon with H-ellipse pores behaves as a marginally stretching-dominated structure with its young's modulus scaling as $E \propto \bar{\rho}^{1.5}$ and strength scaling as $\sigma_{u} \propto \bar{\rho}^{1.5}$ (Figure 6a and 6b). The stretching-dominated signature provides np-Si with the highest values of stiffness, strength, and toughness to weight ratios of all models and complete failure of the structure happens at a strain of $\sim 0.15$ for all relative densities and ligament thicknesses. The architecture of H-ellipse np-Si is accompanied with a highly engaged structure where stress disseminates evenly under tension. In the generic Equations (4) and (5) the proportionality constant $C_{1}$ is less than 1 at a nominal value of 0.92 and the proportionality constant $C_{2}$ is greater than 0.3 at a nominal value of 0.39 for the H-ellipse np-Si.

$$
\begin{gathered}
E=E_{b} C_{1} \bar{\rho}^{n} \\
\sigma_{u}=\sigma_{b} C_{2} \bar{\rho}^{n}
\end{gathered}
$$

np-Si with staggered circular pores exhibits a bending-dominated failure scaling signature with Young's modulus scaling as $E \propto \bar{\rho}^{2}$ and strength scaling as $\sigma_{u} \propto \bar{\rho}^{2}$. In this regime, np-Si's strength, stiffness, and toughness are lowered from that of H-ellipse np-Si by $13.42 \%, 24.32 \%$, and $39.7 \%$ respectively and the strain at failure is lowered to between 0.10 and 0.14 (Figure 5a) depending on relative density and ligament thickness. Structurally, the staggered circle pore geometry carries stress fairly evenly under tension in the $\left[\begin{array}{lll}1 & 0 & 0\end{array}\right]$ direction. In the generic Equations (4) and (5) the proportionality constants $C_{1}$ and $C_{2}$ for np-Si with staggered circles are calculated to be 0.91 and 0.32 respectively. np-Si with V-ellipse pores is found to behave as a 
stochastic structure with young's modulus scaling as $E \propto \bar{\rho}^{2.5}$ and strength scaling as $\sigma_{u} \propto$ $\bar{\rho}^{1.5}$. The strength and stiffness to weight ratios of the V-ellipse np-Si are lower than both the Hellipse models and staggered circles models. However, the toughness is higher than that of the staggered circles np-Si due to a very large strain at failure, between 0.17 and 0.25 . The constants $C_{1}$ and $C_{2}$ for np-Si with $\mathrm{V}$-ellipses are calculated to be 0.51 and 0.21 respectively, which is very low compared to the other models tested here. An Ashby plot in terms of stiffness and material density is provided in Figure 7 to show the comparison between the designed np-Si and known materials. The V-ellipse and H-ellipse np-Si data represent the minimum and maximum values of stiffness from our simulations, with H-ellipse np-Si about twice as stiff as V-ellipse np-Si at high densities. Though, due to H-ellipse np-Si scaling more ideally than V-ellipse np-Si it is $\sim 10$ times stiffer at a relative density of 0.3 and predicted to be $\sim 100$ times stiffer at ultralight relative densities of $\bar{\rho} \sim 0.01$. H-ellipse np-Si scales into a new niche in material property space at densities below $90 \mathrm{~kg} / \mathrm{m}^{3}$ or $\bar{\rho}=0.04$ while other $\mathrm{np}-\mathrm{Si}$ models scale into the existing foam material property space at very low densities.

Toughness $(\kappa)$ is taken as the area under the stress strain curves (Figure 5) from zero strain until ultimate strain at failure and describes how much energy the np-Si models absorb during a full stretch to failure. Models are found to scale as $\kappa \sim \bar{\rho}^{1.5}$ for both H-ellipse and V-ellipse np-Si and $\kappa \sim \bar{\rho}^{2.6}$ for staggered circles np-Si (Figure 6c). Scaling constants, $C_{3}$ in Equation (6), are found to be very low $\sim 0.1$ for each model.

$$
\kappa=\kappa_{b} C_{3} \bar{\rho}^{n}
$$

Interestingly, toughness, stiffness, and strength in $\mathrm{np}-\mathrm{Si}$ are found to be uncoupled each other such that a stronger, stiffer model may not be tougher than its weaker, less stiff counterpart or a 
stronger, tougher model may not be as stiff as its weaker, less tough counterpart. For example, as seen in Figure 6, staggered circle np-Si is stiffer than V-ellipse np-Si for all relative densities, is stronger than V-ellipse np-Si for relative densities greater than 0.44 , and is not as tough as Vellipse np-Si for any relative density below 0.96 .

\subsection{Effect of ligament thickness}

At nanoscale materials usually exhibit size effects such as "smaller is weaker" in nanocrystalline metals[44], "smaller is ductile" in metallic glasses and ceramics[45], and "smaller is stronger" in monocrystalline metals $[5,27,34,46]$. Although the Young's modulus has not been shown, to the best of our knowledge, to be correlated with ligament thickness, strength and toughness have been seen to scale with ligament thickness as a power relationship i.e. $Z(d) \sim c d^{n}$, where $Z$ may represent ultimate strength or toughness, $c$ and $n$ are experimentally determined coefficients, and $d$ is the ligament thickness. No strong, nonlinear curves are found in our data which covers the ligament thickness range of $1.7376 \mathrm{~nm}$ to $13.423 \mathrm{~nm}$ and so we plot our scaling laws for the Young's modulus, ultimate strength, and toughness of np-Si linearly as $Z(d)=b d+c$.

Young's modulus, ultimate strength, and toughness are calculated from the stress-strain curves in Figure 8 and plotted in Figures 9a, 9b, and 9c. The linear region of each stress-strain curve is taken to measure the Young's modulus, the peak of each curve is taken as the ultimate strength, and the area under each curve from zero strain until the strain at failure is taken as the toughness. Interestingly, H-ellipse np-Si behaves unlike staggered circles np-Si and V-ellipse np-Si for all three attributes (strength, toughness, and ductility) with respect to ligament thickness. In the case of np-Si with V-ellipses and staggered circles the relationships "smaller is stronger" and "smaller is ductile" are observed. Under constant relative density, our simulations predict that np-Si with 
V-ellipses gains $170 \mathrm{MPa}$ in ultimate strength for every nanometer that the average ligament thickness is reduced and np-Si with staggered circular pores gains $105 \mathrm{MPa}$ per nanometer reduction in ligament thickness. However, the simulations show that np-Si can lose $\sim 100 \mathrm{MPa}$ in ultimate strength for every nanometer that the average ligament thickness is reduced if the pore geometry is changed to H-ellipse np-Si. As ligament diameter approaches zero the strain at failure for V-ellipse np-Si approaches 0.227 while the strain at failure for staggered circles approaches 0.149 . However, in the case of H-ellipse np-Si smaller is neither stronger nor more ductile. Strength decreases as ligament thickness decreases and ductility does not change very obviously as ligament thickness changes. In sum, from Figure 9 it is clear to show that size effects exist for ultimate strength, toughness, and ultimate strain while for the Young's modulus the size effect can be negligible.

\section{Conclusions}

Our observations show that the scaling laws of np-Si are dependent on pore geometry, pore orientation, relative density, and ligament thickness. Changing pore geometry and pore orientation can affect the stress distribution within the np-Si structures by increasing or decreasing the number of atoms available to carry the load; this in turn directly leads to changes in the mechanical properties of $\mathrm{np}-\mathrm{Si}$. Decreasing relative density results in decreases in all mechanical properties where: Young's modulus scales as $E \propto \rho^{n}$ with $\mathrm{n}$ ranging from an almost stretching-dominated signature of 1.5 to a stochastic signature of 2.5 , strength scales as $\sigma_{u} \propto \rho^{n}$ with $n$ ranging from 1.5 to 2 , and toughness scales as $\kappa \propto \rho^{n}$ with $n$ ranging from 1.5 to 2.6. Toughness in np-Si is found to be uncoupled from Young's modulus and strength such that a stronger, stiffer model may not be tougher than its weaker, less stiff counterpart. Our findings also show that ligament thickness plays a negligible effect on the Young's modulus of np-Si in 
the range of $1.7 \mathrm{~nm}$ to $13.4 \mathrm{~nm}$; however trends are strong enough to report for ultimate strength, toughness, and ductility. Size effects are not consistent for np-Si models with different nanostructures: np-Si with staggered circular pores and vertical elliptical pores become stronger and more ductile as ligament thickness decreases while np-Si with horizontal elliptical pores becomes weaker as ligament thickness decreases and shows no discernable change in ductility as ligament thickness changes. These results promise much for the design of nanoporous silicon structures with tunable, desirable mechanical properties stemming from their nanoscopic structure, paving the way for a new class of porous materials.

\section{Author Information}

Corresponding authors: Xianqiao Wang (qwwang@uga.edu)

Notes: The authors declare no competing financial interest.

\section{Acknowledgement}

The authors acknowledge support from the National Science Foundation and University of Georgia (UGA) Research Foundation. Calculations are performed at the UGA Advanced Computing Resource Centre.

\section{References}


[1] K.A. Shaw, Z.L. Zhang, N.C. MacDonald. SCREAM I: A single mask, single-crystal silicon, reactive ion etching process for microelectromechanical structures, Sensors and Actuators A: Physical 40 (1994) 63-70.

[2] M. Shi, H. Tang, X. Shao, X. Huang, G. Cao, R. Wang, T. Li, X. Li, H. Gong. Interface property of silicon nitride films grown by inductively coupled plasma chemical vapor deposition and plasma enhanced chemical vapor deposition on In0.82Al0.18As, Infrared Physics \& Technology 71 (2015) 384388.

[3] J.A. Dagata, J. Schneir, H.H. Harary, C.J. Evans, M.T. Postek, J. Bennett. Modification of hydrogen-passivated silicon by a scanning tunneling microscope operating in air, Applied Physics Letters 56 (1990) 2001-2003.

[4] A. Wittstock, V. Zielasek, J. Biener, C.M. Friend, M. Bäumer. Nanoporous Gold Catalysts for Selective Gas-Phase Oxidative Coupling of Methanol at Low Temperature, Science 327 (2010) 319-322.

[5] J. Biener, A.M. Hodge, A.V. Hamza, L.M. Hsiung, J.H. Satcher. Nanoporous Au: A high yield strength material, Journal of Applied Physics 97 (2005) 024301.

[6] E. Detsi, Z.G. Chen, W.P. Vellinga, P.R. Onck, J.T.M. De Hosson. Reversible strain by physisorption in nanoporous gold, Applied Physics Letters 99 (2011) 083104.

[7] N. Zehfroosh, M. Shahmohammadi, S. Mohajerzadeh. High-Sensitivity lon-Selective Field-Effect Transistors Using Nanoporous Silicon, Electron Device Letters, IEEE 31 (2010) 1056-1058.

[8] S.D. Alvarez, A.M. Derfus, M.P. Schwartz, S.N. Bhatia, M.J. Sailor. The compatibility of hepatocytes with chemically modified porous silicon with reference to in vitro biosensors, Biomaterials 30 (2009) 26-34.

[9] W. Churaman, L. Currano, C. Becker. Initiation and reaction tuning of nanoporous energetic silicon, Journal of Physics and Chemistry of Solids 71 (2010) 69-74.

[10] J. Germain, J.M.J. Frechet, F. Svec. Hypercrosslinked polyanilines with nanoporous structure and high surface area: potential adsorbents for hydrogen storage, Journal of Materials Chemistry 17 (2007) 4989-4997.

[11] J. Parkinson, R. Gordon. Beyond micromachining: the potential of diatoms, Trends in Biotechnology 17 (1999) 190-196.

[12] F.E. Round, R.M. Crawford, D.G. Mann. Diatoms: biology and morphology of the genera, Cambridge University Press, 1990.

[13] C.E. Hamm, R. Merkel, O. Springer, P. Jurkojc, C. Maier, K. Prechtel, V. Smetacek. Architecture and material properties of diatom shells provide effective mechanical protection, Nature 421 (2003) 841-843.

[14] C.-Y. Chen, H.-X. Li, M.E. Davis. Studies on mesoporous materials, Microporous Materials 2 (1993) 17-26.

[15] A. Ressine, S. Ekström, G. Marko-Varga, T. Laurell. Macro-/Nanoporous Silicon as a Support for High-Performance Protein Microarrays, Analytical Chemistry 75 (2003) 6968-6974.

[16] T. Wada, T. Ichitsubo, K. Yubuta, H. Segawa, H. Yoshida, H. Kato. Bulk-Nanoporous-Silicon Negative Electrode with Extremely High Cyclability for Lithium-Ion Batteries Prepared Using a Top-Down Process, Nano Letters 14 (2014) 4505-4510.

[17] G. Wang, Y. Yang, J.-H. Lee, V. Abramova, H. Fei, G. Ruan, E.L. Thomas, J.M. Tour. Nanoporous Silicon Oxide Memory, Nano letters 14 (2014) 4694-4699.

[18] A.P. Garcia, D. Sen, M.J. Buehler. Hierarchical Silica Nanostructures Inspired by Diatom Algae Yield Superior Deformability, Toughness, and Strength, Metallurgical and Materials Transactions A 42 (2011) 3889-3897.

[19] D. Sen, A.P. Garcia, M.J. Buehler. Mechanics of Nano-Honeycomb Silica Structures: SizeDependent Brittle-to-Ductile Transition, Journal of Nanomechanics and Micromechanics 1 (2011) 112118. 
[20] A.P. Garcia, M.J. Buehler. Bioinspired nanoporous silicon provides great toughness at great deformability, Computational Materials Science 48 (2010) 303-309.

[21] S. Plimpton. Fast Parallel Algorithms for Short-Range Molecular Dynamics, Journal of Computational Physics 117 (1995) 1-19.

[22] J.F. Justo, M.Z. Bazant, E. Kaxiras, V.V. Bulatov, S. Yip. Interatomic potential for silicon defects and disordered phases, Physical Review B 58 (1998) 2539-2550.

[23] F.H. Stillinger, T.A. Weber. Computer simulation of local order in condensed phases of silicon, Physical Review B 31 (1985) 5262-5271.

[24] J. Tersoff. New empirical approach for the structure and energy of covalent systems, Physical Review B 37 (1988) 6991-7000.

[25] M.A. Hopcroft, W.D. Nix, T.W. Kenny. What is the Young's Modulus of Silicon?, Microelectromechanical Systems, Journal of 19 (2010) 229-238.

[26] F. Montagne, N. Blondiaux, A. Bojko, R. Pugin. Molecular transport through nanoporous silicon nitride membranes produced from self-assembling block copolymers, Nanoscale 4 (2012) 5880-5886.

[27] J. Biener, A.M. Hodge, J.R. Hayes, C.A. Volkert, L.A. Zepeda-Ruiz, A.V. Hamza, F.F. Abraham. Size Effects on the Mechanical Behavior of Nanoporous Au, Nano Letters 6 (2006) 2379-2382.

[28] N. Winter, M. Becton, L. Zhang, X. Wang. Failure Mechanisms and Scaling Laws of Nanoporous Aluminum: A Computational Study Advanced Engineering Materials (2015) 632-642.

[29] A. Stuckowski. Visualization and and analysis of atomistic simulation data with OVITO - open visualization tool, Modelling and Simulation in Materials Science and Engineering 18 (2010).

[30] L. Moroni, J.R. de Wijn, C.A. van Blitterswijk. 3D fiber-deposited scaffolds for tissue engineering: Influence of pores geometry and architecture on dynamic mechanical properties, Biomaterials 27 (2006) 974-985.

[31] D.-M. Liu. Control of pore geometry on influencing the mechanical property of porous hydroxyapatite bioceramic, J Mater Sci Lett 15 (1996) 419-421.

[32] L.R. Meza, S. Das, J.R. Greer. Strong, lightweight, and recoverable three-dimensional ceramic nanolattices, Science 345 (2014) 1322-1326.

[33] C.A. Volkert, E.T. Lilleodden. Size effects in the deformation of sub-micron Au columns, Philosophical Magazine 86 (2006) 5567-5579.

[34] X.-Y. Sun, G.-K. Xu, X. Li, X.-Q. Feng, H. Gao. Mechanical properties and scaling laws of nanoporous gold, Journal of Applied Physics 113 (2013) 023505.

[35] J.F. Sadoc, N. Rivier. Foams and Emulsions, Springer Netherlands, 2013.

[36] A.H. Chokshi, A. Rosen, J. Karch, H. Gleiter. On the validity of the hall-petch relationship in nanocrystalline materials, Scripta Metallurgica 23 (1989) 1679-1683.

[37] T.G. Nieh, J. Wadsworth. Hall-petch relation in nanocrystalline solids, Scripta Metallurgica et Materialia 25 (1991) 955-958.

[38] M. Becton, X. Wang. Grain-size dependence of mechanical properties in polycrystalline boronnitride: a computational study, Physical Chemistry Chemical Physics 17 (2015) 21894-21901.

[39] A.M. Hodge, J. Biener, J.R. Hayes, P.M. Bythrow, C.A. Volkert, A.V. Hamza. Scaling equation for yield strength of nanoporous open-cell foams, Acta Materialia 55 (2007) 1343-1349.

[40] V.S. Deshpande, N.A. Fleck, M.F. Ashby. Effective properties of the octet-truss lattice material, Journal of the Mechanics and Physics of Solids 49 (2001) 1747-1769.

[41] T.A. Schaedler, A.J. Jacobsen, A. Torrents, A.E. Sorensen, J. Lian, J.R. Greer, L. Valdevit, W.B. Carter. Ultralight metallic microlattices, Science 334 (2011) 962-965.

[42] N. Fleck, V. Deshpande, M. Ashby. Micro-architectured materials: past, present and future. Proceedings of the Royal Society of London A: Mathematical, Physical and Engineering Sciences, vol. 466: The Royal Society, 2010. p.2495-2516. 
[43] L. Montemayor, J. Greer. Mechanical Response of Hollow Metallic Nanolattices: Combining Structural and Material Size Effects, Journal of Applied Mechanics 82 (2015) 071012.

[44] J.R. Greer, D. Jang, X.W. Gu. Exploring Deformation Mechanisms in Nanostructured Materials, JOM 64 (2012) 1241-1252.

[45] C.Q. Chen, Y.T. Pei, J.T.M. De Hosson. Effects of size on the mechanical response of metallic glasses investigated through in situ TEM bending and compression experiments, Acta Materialia 58 (2010) 189-200.

[46] B. Reischl, A. Kuronen, K. Nordlund. Nanoindentation of gold nanorods with an atomic force microscope, Materials Research Express 1 (2014) 045042. 
(a)

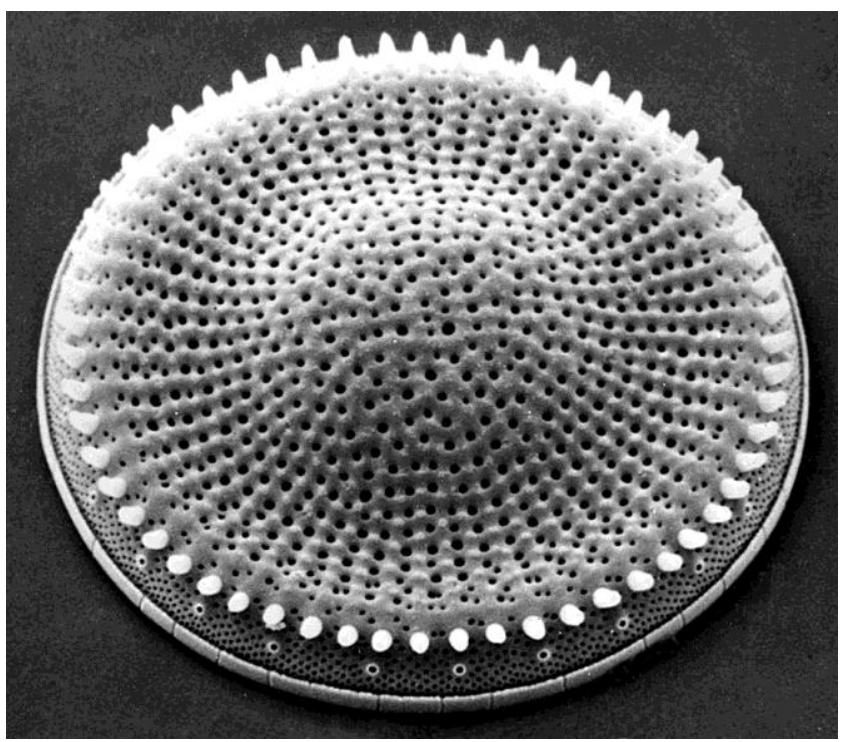

(b)

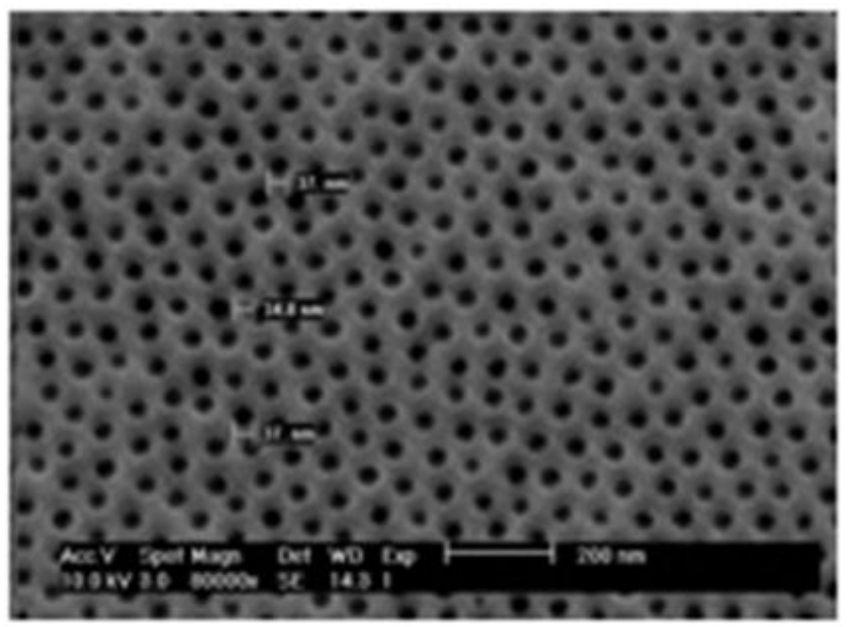

(c)

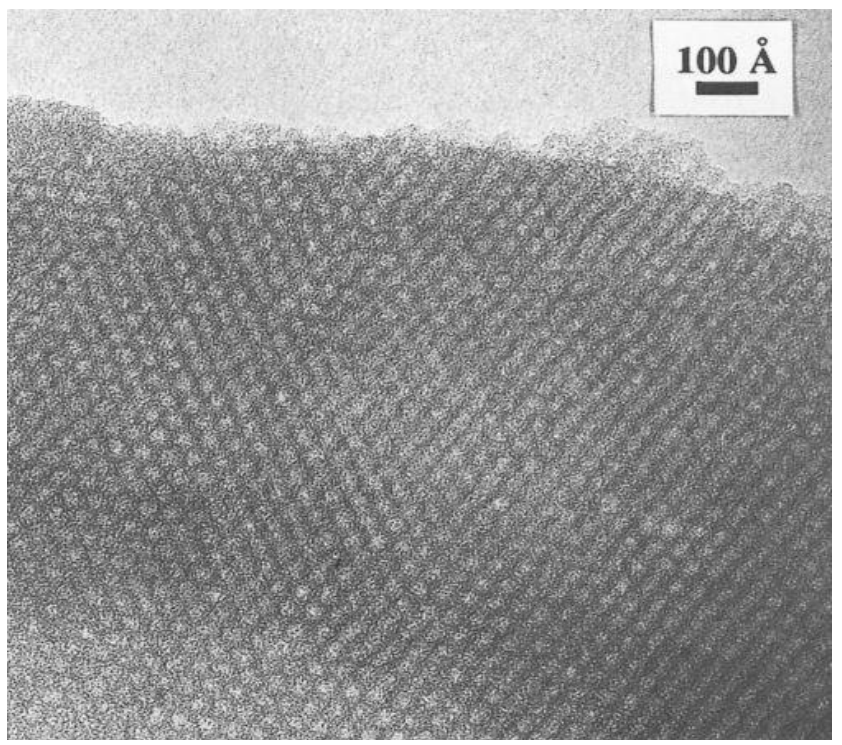

Figure 1: (a) Structure of diatoms, showing their porous silica structure (image is reprinted from Ref [12] with permission); (b) a TEM picture of np-Si produced via lithography (image is reprinted from Ref. [23] with permission) (c) TEM photograph of calcined np-Si known as MCM-41 (image is reprinted from Ref. [14] with permission). 
(a)

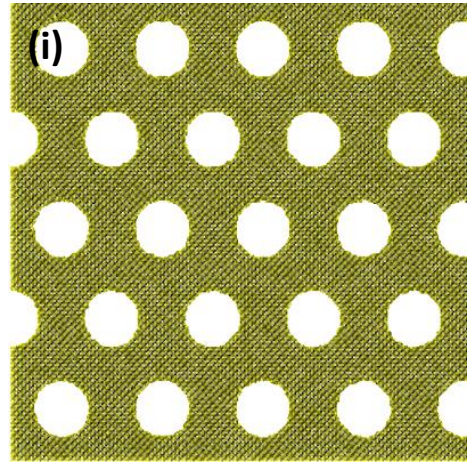

(b)

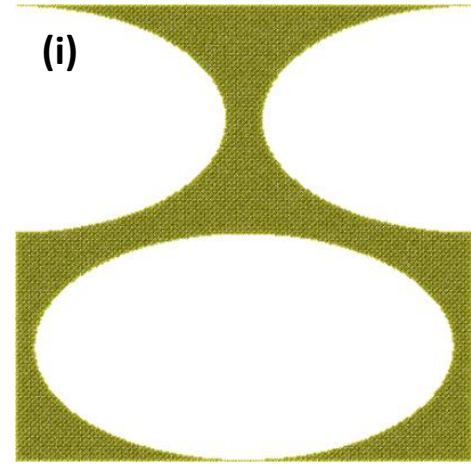

(c)

\section{(ii)}

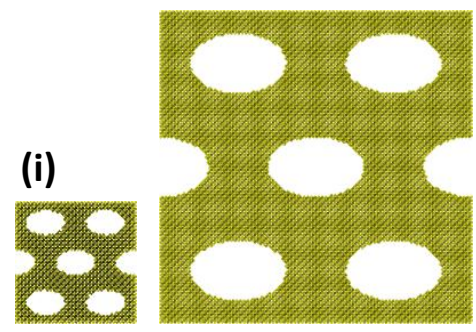

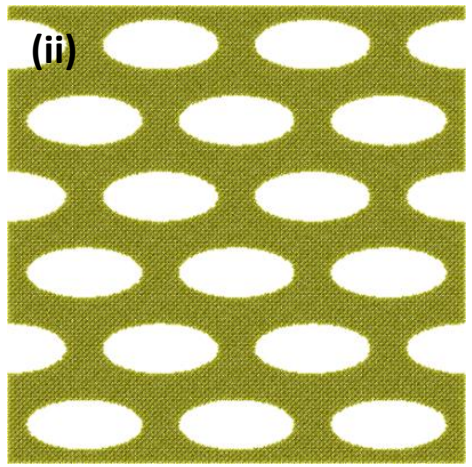
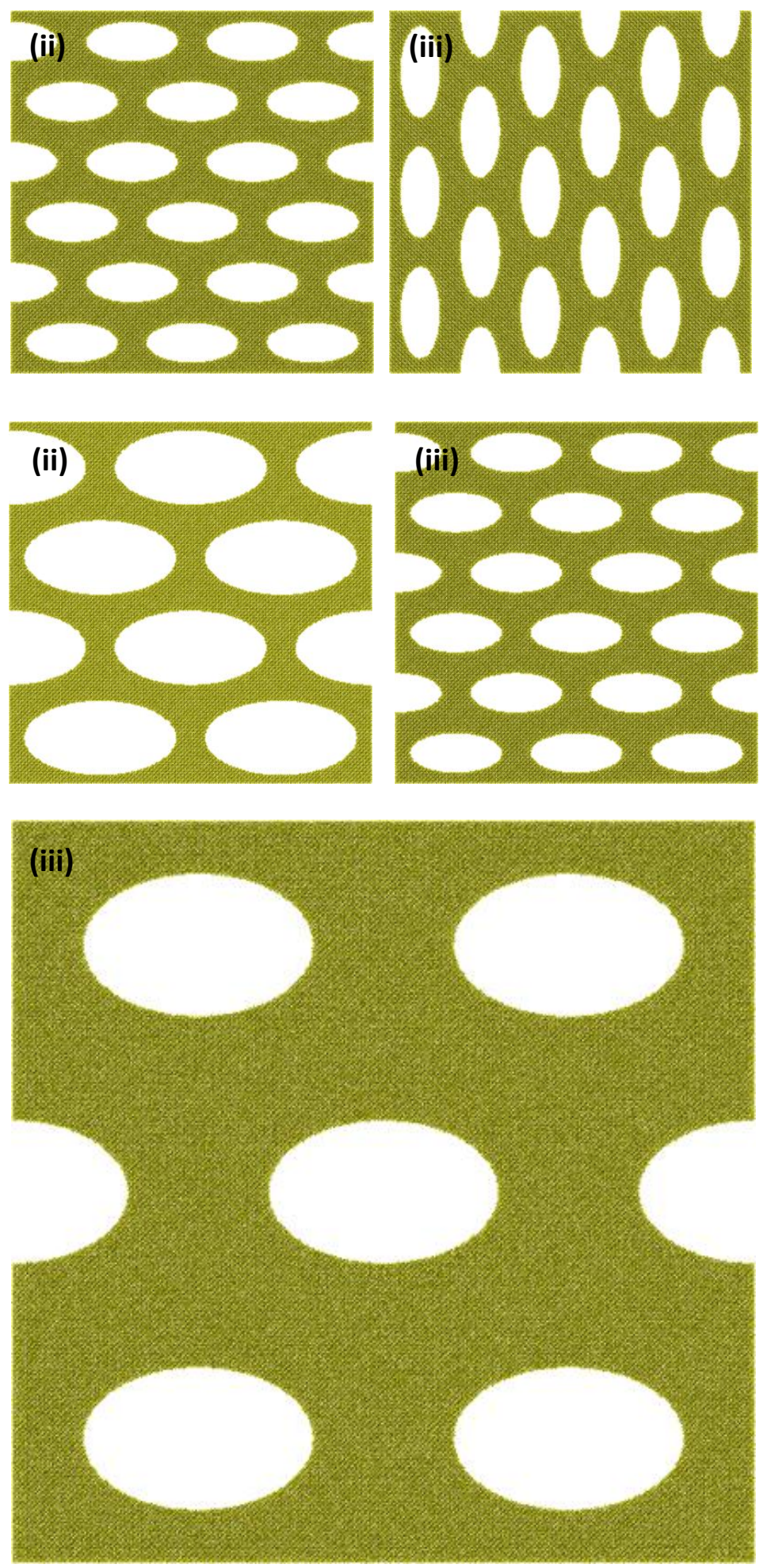

Figure 2: (a) np-Si with ligand thicknesses of $3.5 \mathrm{~nm}$ (i) Staggered Circles (SC) (ii) Horizontal Ellipses (H-ellipses) and (iii) Vertical Ellipses (V-ellipses); (b) H-ellipse np-Si with ligament thicknesses of $3.5 \mathrm{~nm}$ and relative densities of (i) 0.313 (ii) 0.495 and (iii) 0.67. (c) H-ellipse np-Si with relative densities of 0.71 and ligament thicknesses of (i) $1.74 \mathrm{~nm}$ (ii) $4.47 \mathrm{~nm}$ and (iii) $13.42 \mathrm{~nm}$. Snapshots in row (c) are shown to scale with respect to each other but not with respect to snapshots in rows (a) or (b). 
(a)

(i)

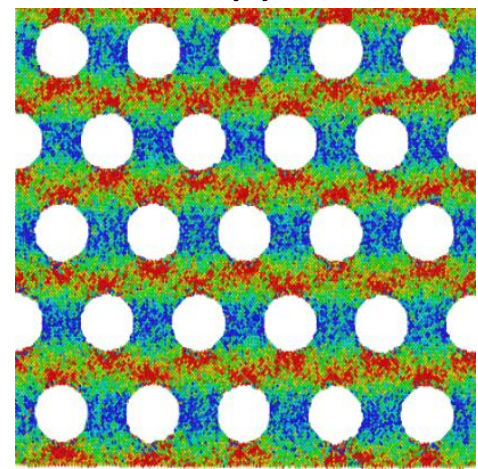

(ii)

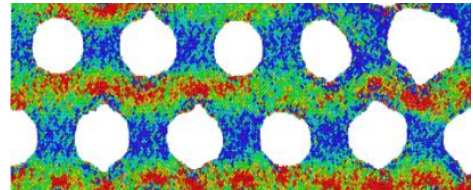

(iii)
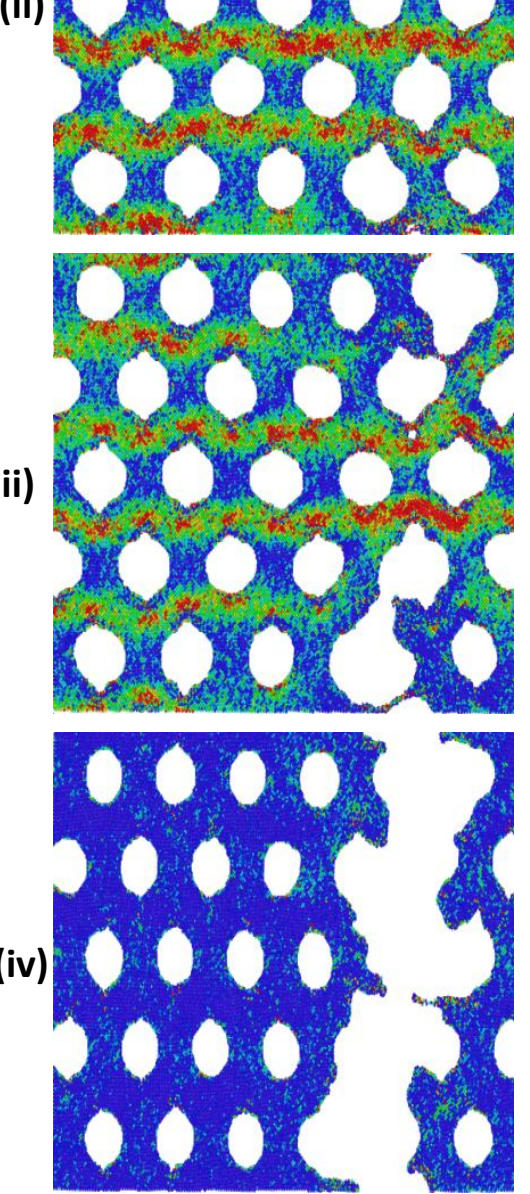

per atom stress $\left(\mathrm{GPa}^{*} \mathrm{~nm}^{\wedge} 3\right)$

0.00 (b)
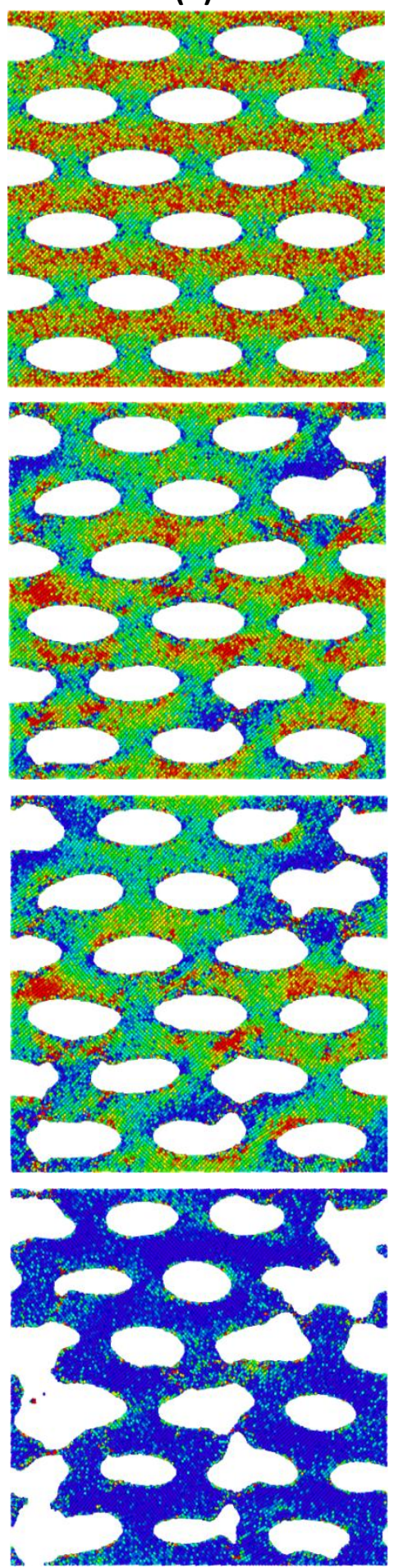

0.35 (c)
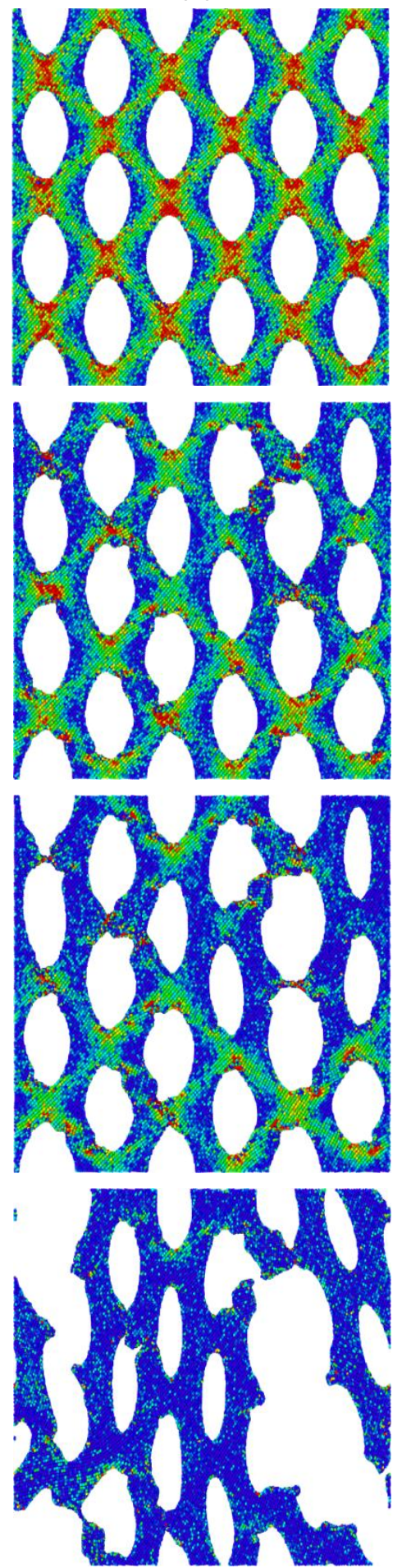

Figure 3: Frame by frame failure of np-Si having different pore geometries/orientations.

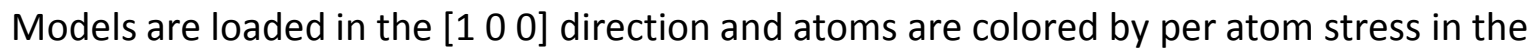
$\left[\begin{array}{lll}1 & 0 & 0\end{array}\right]$ direction. (a) Np-Si with staggered circular pores (row (i) strain of 0.11). (b) Np-si with $\mathrm{H}$-ellipse pore geometry (row (i) strain of 0.13 ). (c) Np-Si with V-ellipse geometry (row (i) strain of 0.16). Snapshots begin at peak stress/strain and end when stress reached zero. 

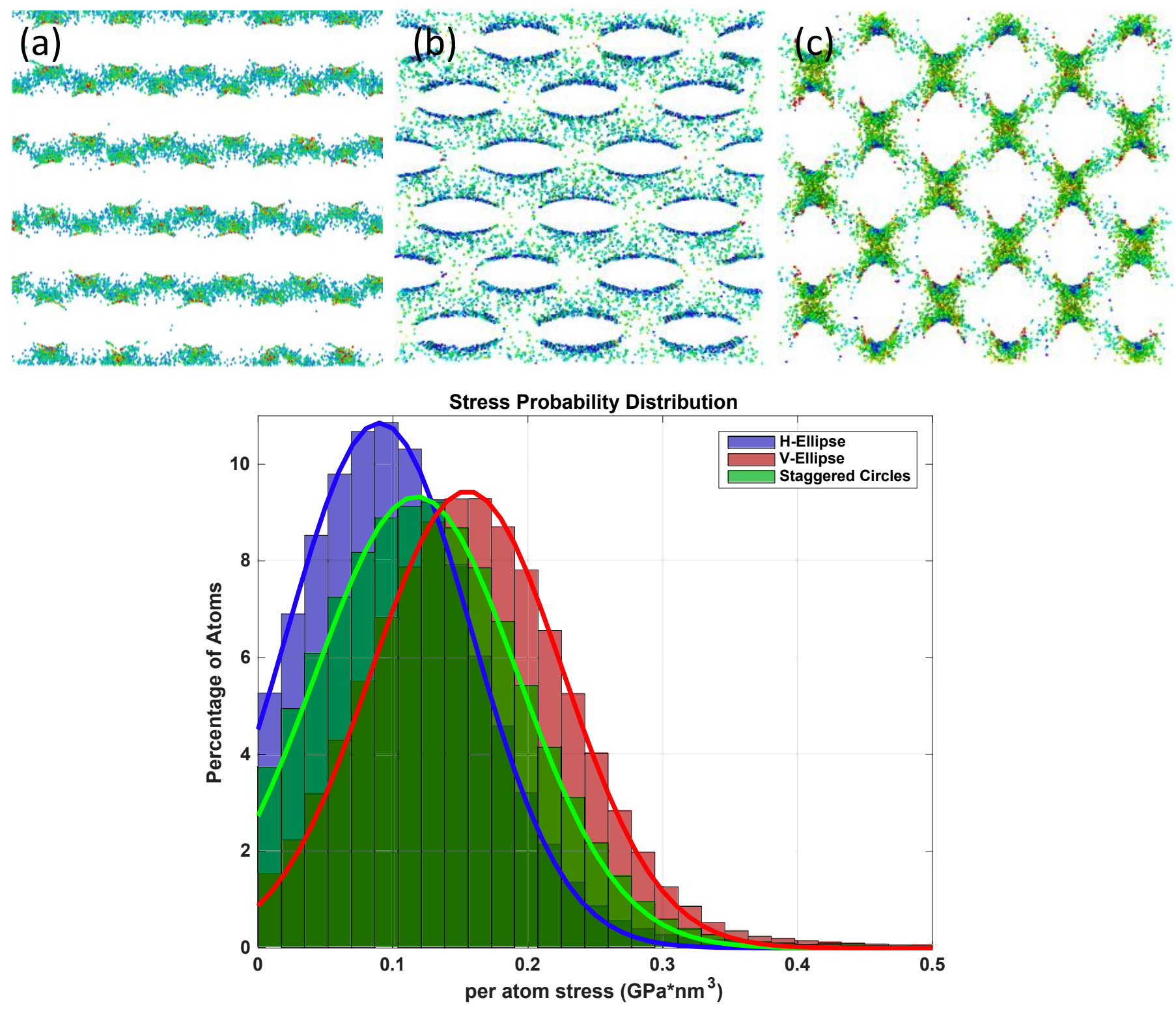

Figure 4: snapshots corresponding to figure 3 row (i), where only atoms experiencing a per atom stress of $0.35 \mathrm{GPa} \times \mathrm{nm}^{3}$ or higher are shown. (a) Staggered Circles Np-Si (b) H-Ellipse Np-Si (c) $\mathrm{V}$-Ellipse $\mathrm{Np}$-Si (d) histogram showing the percentage of atoms experiencing a per atom stress between 0 and $0.5 \mathrm{GPa} \times \mathrm{nm}^{3}$ 

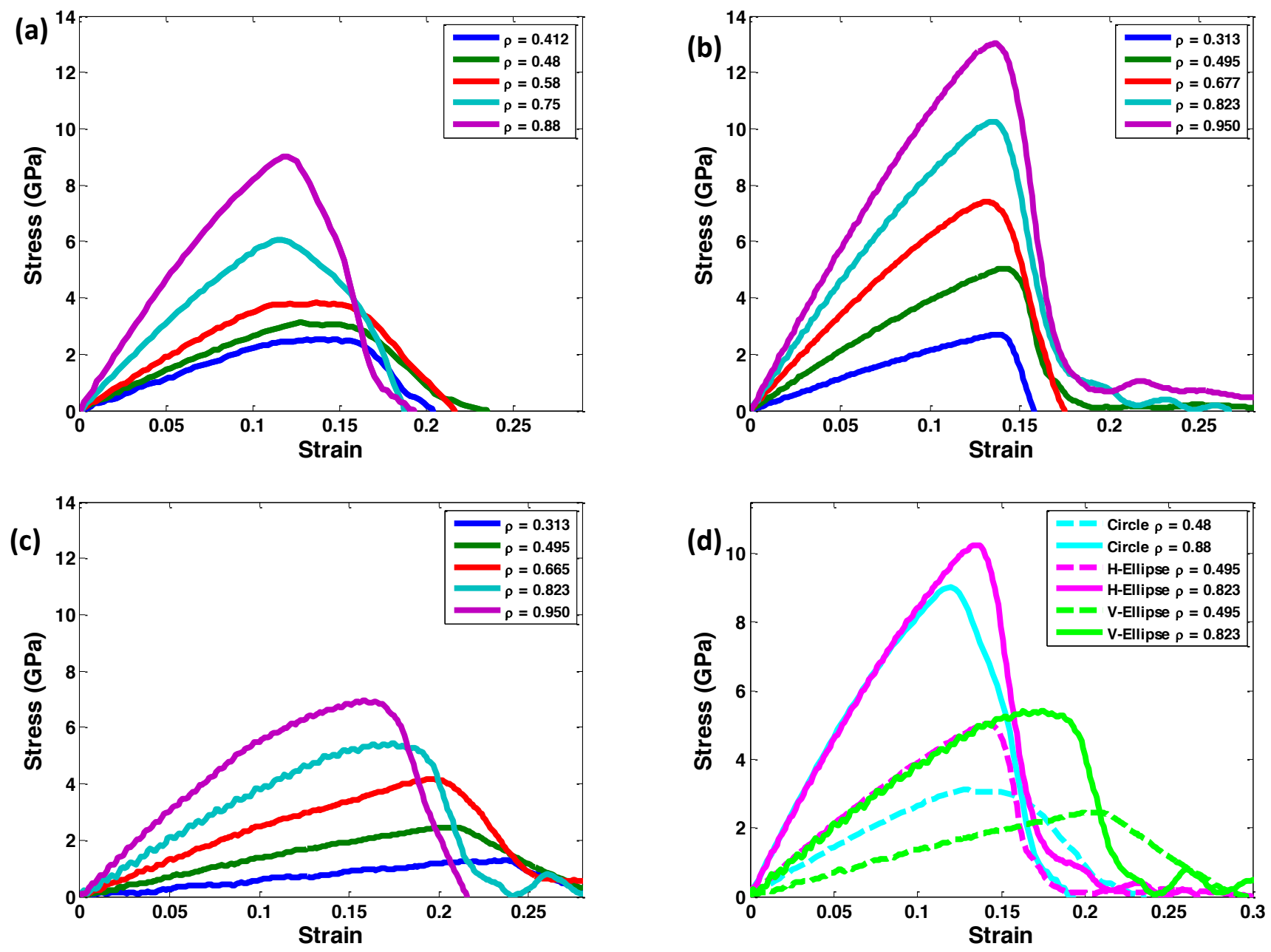

Figure 5: Stress-strain relationship of $\mathrm{np}$-Si versus the relative density under three different pore patterns: (a) staggered circles; (b) staggered horizontal ellipses; (c) staggered vertical ellipses; (d) a comparison among these three cases. Ligament thickness $=3.5 \mathrm{~nm}$. 

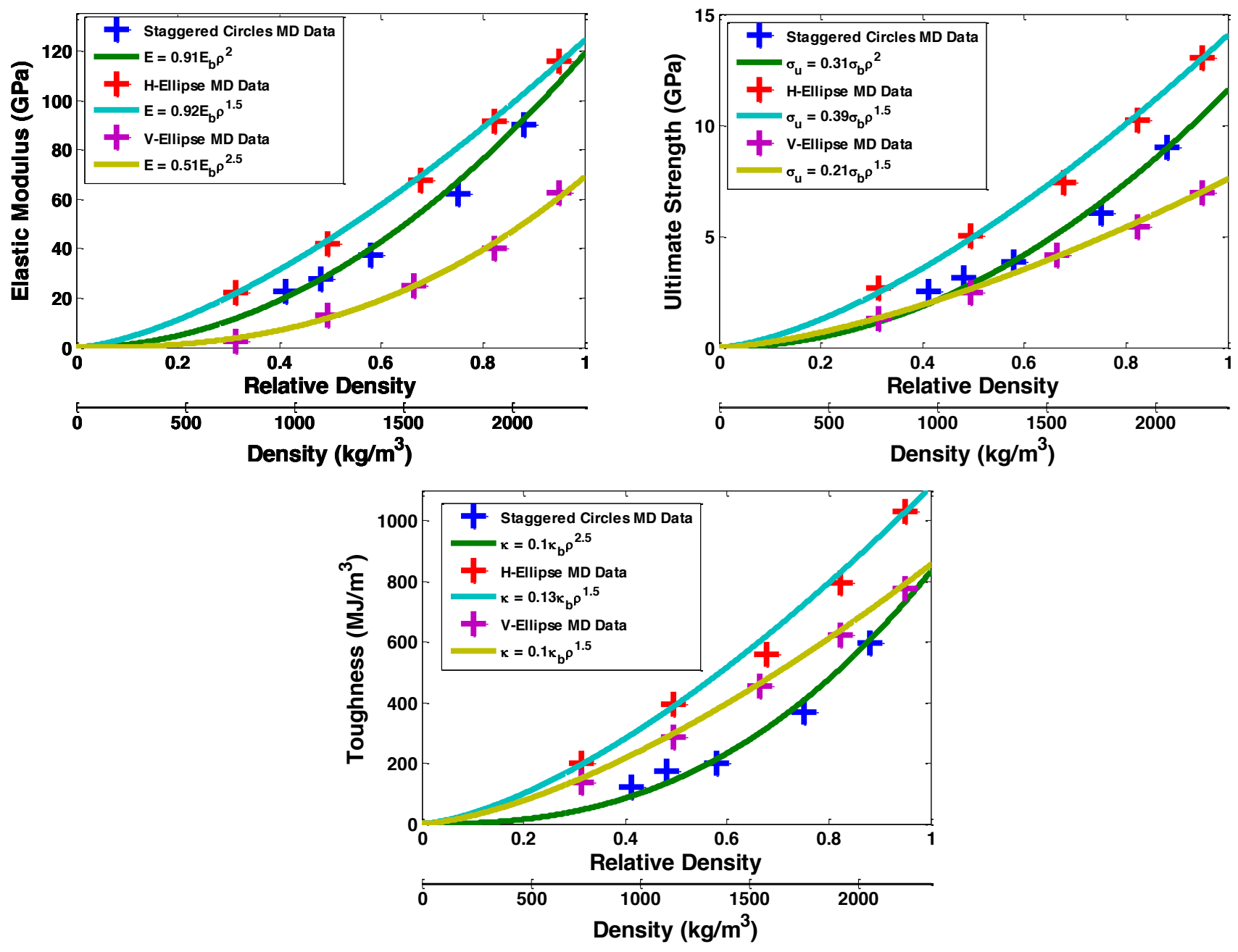

Figure 6: Semi-empirical correlations of material properties versus relative density. (a) elastic modulus; (b) ultimate strength; (c) toughness. Cross-hairs represent MD data and is fitted by a scaling curve. Ligament thickness $=3.5 \mathrm{~nm}$. 


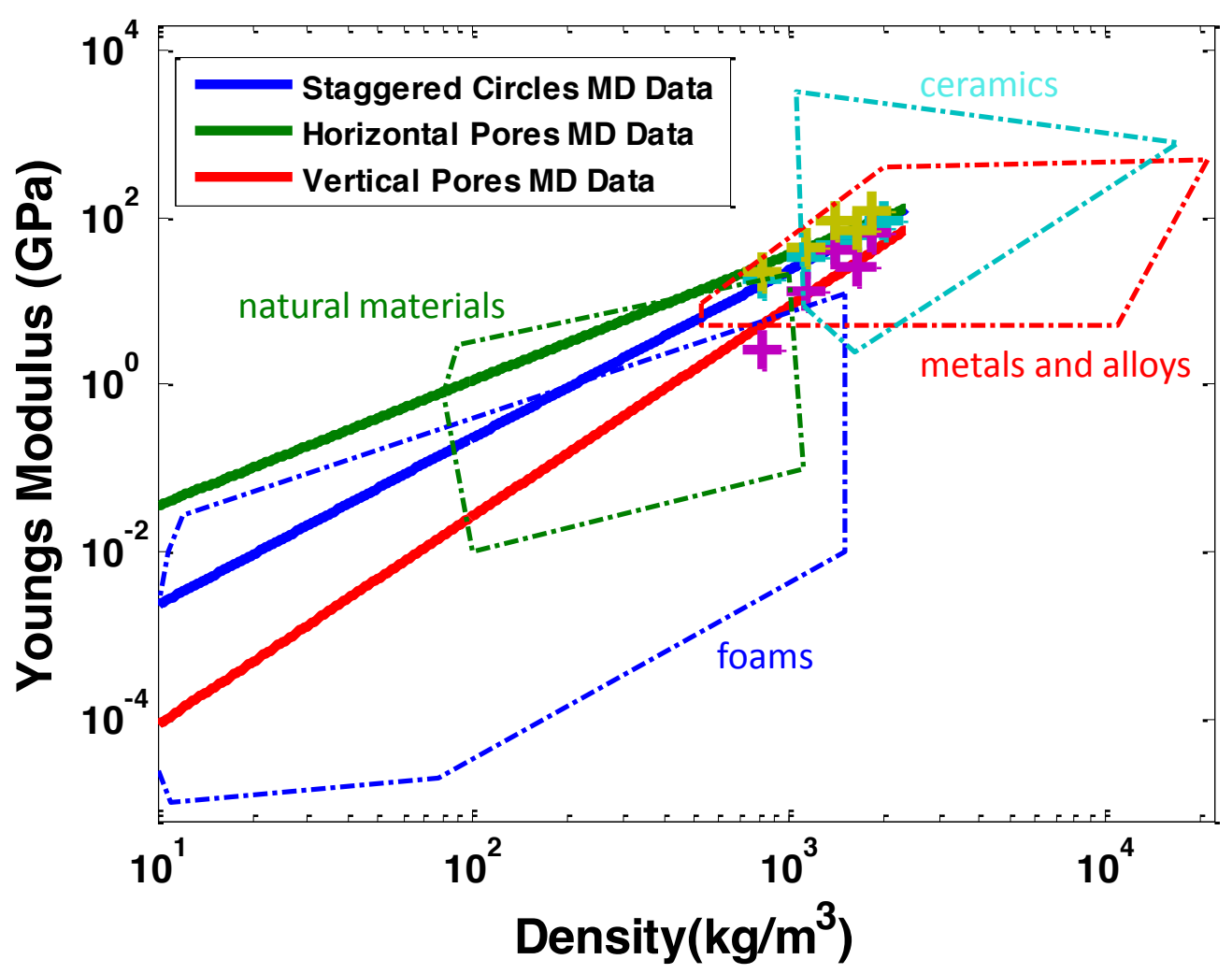

Figure 7: Material property space plot of Young's modulus versus density, with dotted regions showing the phase space of common structural materials. 

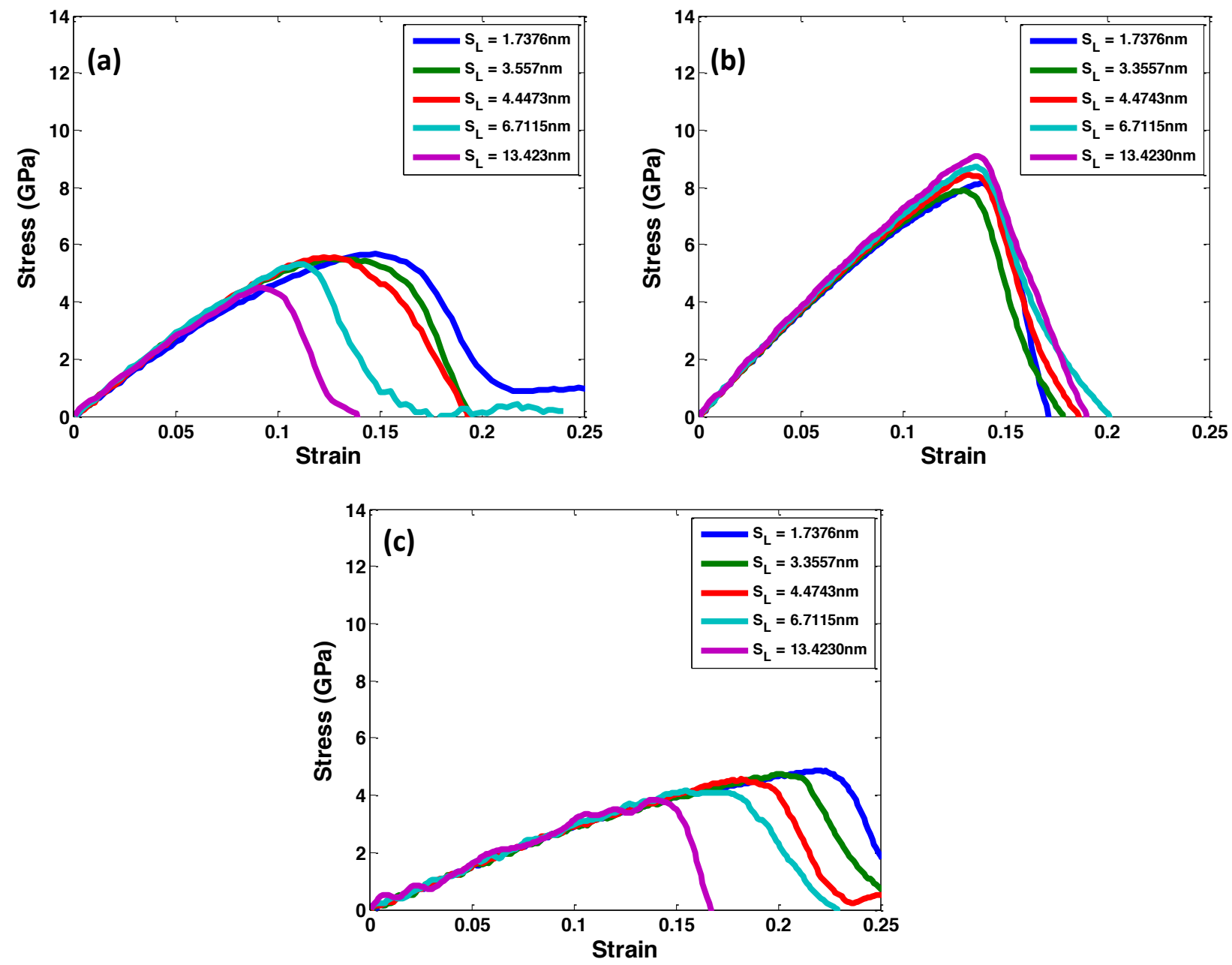

Figure 8: Stress-strain relationship of $\mathrm{np}$-Si versus the ligament size under the same relative density: (a) staggered circles; (b) staggered horizontal ellipses; (c) staggered vertical ellipses. Relative density $=3.5 \mathrm{~nm}$. 

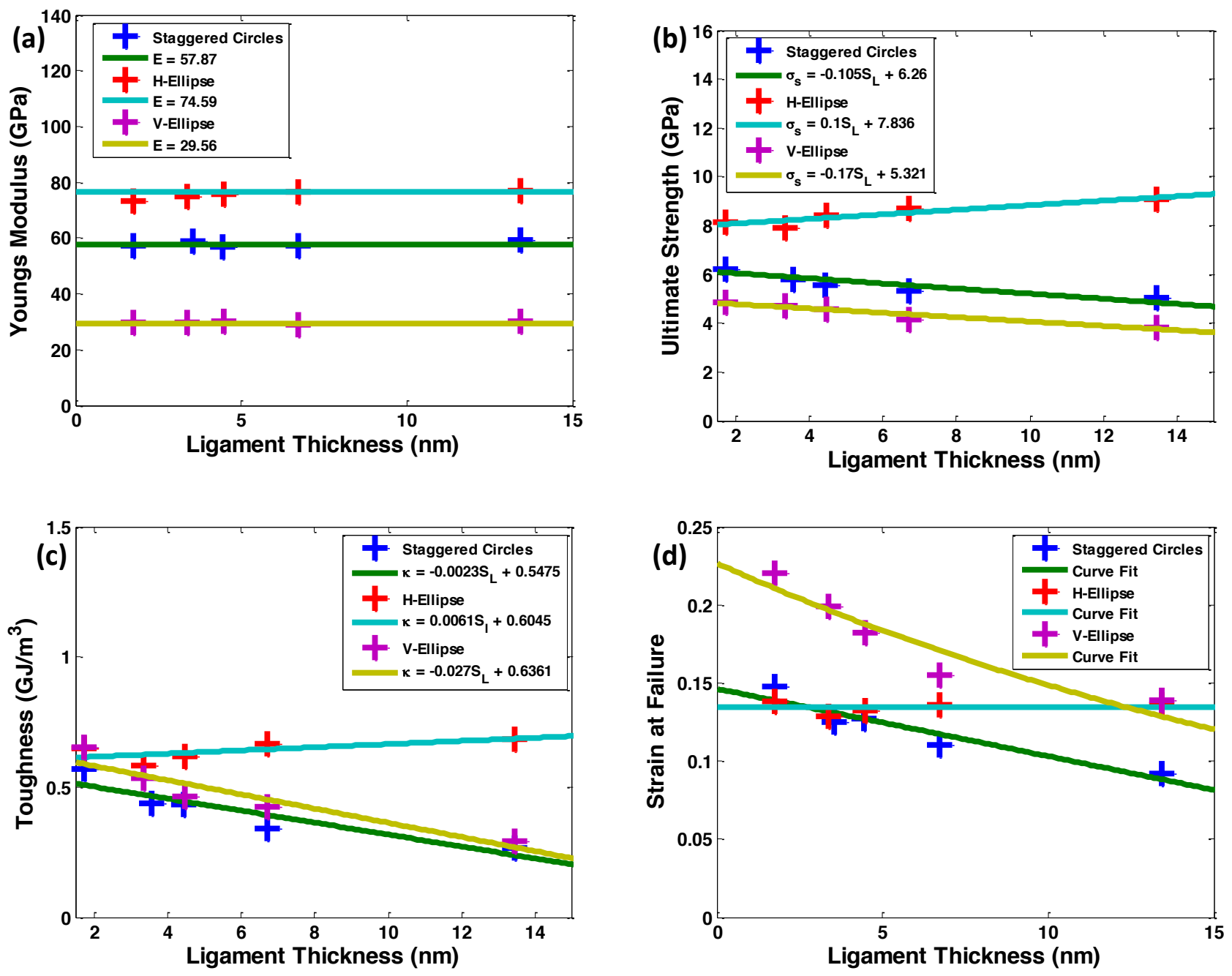

Figure 9: Semi-empirical correlations of materials properties versus the ligament size.: (a) elastic modulus; (b) ultimate strength; (c) toughness. (d) strain at ultimate stress/failure as a function of ligament thickness. Relative density is around 0.71 . 
Table 1: Comparison of mechanical properties for $\mathrm{np}$-Si with different pore geometry

\begin{tabular}{|l|c|c|c|}
\hline Pore Geometry & $\begin{array}{l}\text { Young's Modulus } \\
(\mathrm{GPa})\end{array}$ & $\begin{array}{l}\text { Ultimate Strength } \\
(\mathrm{GPa})\end{array}$ & $\begin{array}{l}\text { Toughness } \\
\left(\mathrm{MJ} / \mathrm{m}^{3}\right)\end{array}$ \\
\hline H-ellipses & 67.8 & 7.4 & 557.8 \\
\hline Staggered circles & 58.7 & 5.6 & 336.4 \\
\hline V-ellipses & 25.0 & 4.2 & 453.4 \\
\hline
\end{tabular}




\section{Effects of Pore Design on Mechanical Properties of Nanoporous Silicon}

Nicholas Winter, Matthew Becton, Liuyang Zhang, and Xianqiao Wang* College of Engineering, University of Georgia, Athens, GA 30602

*Corresponding author: xqwang@uga.edu

Molecular dynamics simulations are performed to study the effects of ligament thickness, relative density, and pore geometry/orientation on the mechanical properties of nanoporous silicon, thereby determining its Young's modulus, ultimate strength, and toughness as well as the scaling trends versus the features of interior ligaments.
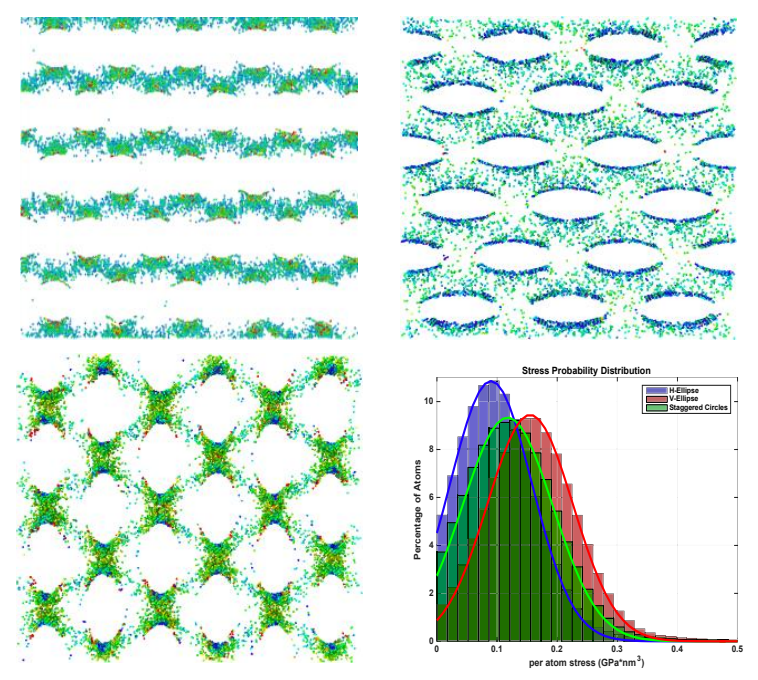